\title{
INVESTIGACIONES GEOGRÁFICAS
}

INSTITUTO UNIVERSITARIO DE GEOGRAFÍA

UNIVERSIDAD DE ALICANTE

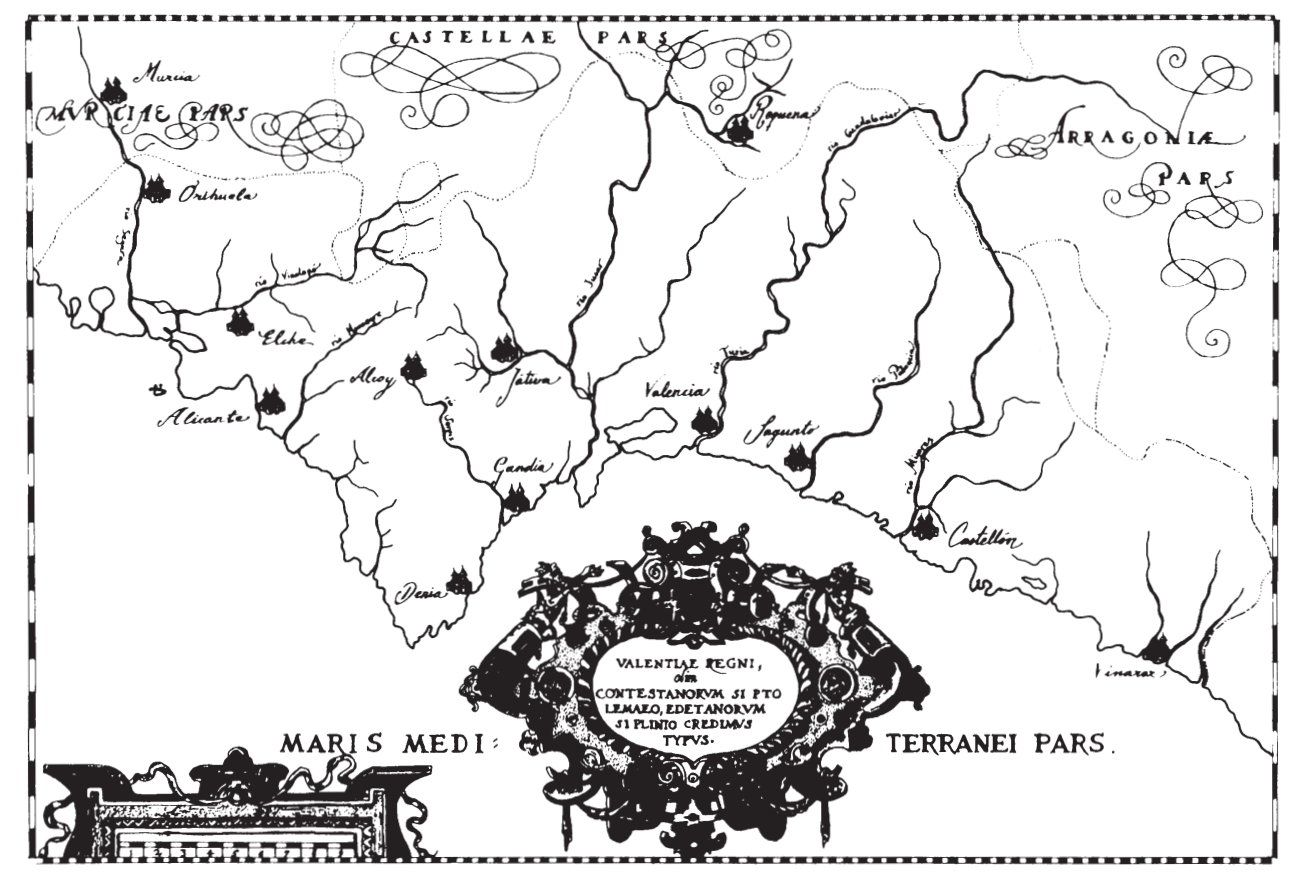

ANALES DE LA UNIVERSIDAD DE ALICANTE INSTITUTO UNIVERSITARIO DE GEOGRAFÍA ENERO-ABRIL 2003 


\section{DARIO CROCE (1939-2002)}

El domingo 8 de diciembre falleció, tras una breve enfermedad, el profesor Dario Croce, miembro del Consejo Asesor de la revista Investigaciones Geográficas y uno de los más esclarecidos geógrafos italianos, como corrobora su sólida y dilatada curricula científica, resultado de cuarenta años de dedicación a la docencia y a la investigación.

Licenciado en Ciencias Geológicas en 1963 por la Università degli Studi di Padova, su vinculación a la Geografía se inició con anterioridad a la conclusión de sus estudios al cursar diversas asignaturas impartidas por G. Morandini, uno de los maestros que institucionalizaron la Geografía en Italia en la primera mitad del siglo XX. Esta inicial relación con el saber geográfico fue progresivamente consolidándose como resultado de sus iniciales líneas de investigación, las relaciones entre la sociedad y el medio, y por su actividad docente al ejercer como profesor ayudante (Assistente Volontario) de 1963 a 1965 en la cátedra de Geografía de la Facoltà di Magistero. Su labor docente en dicho Centro se prolongó hasta su fallecimiento, ocupando diferentes puestos (Asistente Incaricato, Asistente di Ruolo, Incaricato Stabilizzato, Professore Associato, Profesore Straordinario), hasta lograr el grado de Catedrático de Universidad (Profesore Ordinario) en 1986.

$\mathrm{Su}$ actividad científica se centró en diversas ramas de la Geografía, si bien evidenció una marcada preferencia por aquéllas que hacen referencia al análisis de los paisajes, a las relaciones entre la sociedad y el medio, a la gestión de los recursos hídricos, a la Geografía Rural y concretamente el análisis de las estructuras agrarias y los regadíos tanto italianos (llanura y delta del Po) como españoles (depresión del Ebro) y a los procesos de territorialización en los países áridos y especialmente en África, en el ámbito de las investigaciones desarrolladas por el grupo de trabajo de la UGI Resource, management in drylands.

En el desarrollo de las citadas líneas de investigación, el trabajo de campo fue siempre una constante, a la que otorgaba una enorme relevancia para un adecuado conocimiento de los paisajes —entendidos como resultado de las relaciones entre una sociedad y el medio donde ésta se asienta-, y los procesos de ordenación y gestión territorial. El interés por otro de los métodos de la Geografía, el análisis escalar y comparativo, y concretamente la comparación entre los regadíos españoles e italianos, explica su relación con la comunidad universitaria española, que se inicia, en una primera etapa, en la Universidad de Zaragoza, donde desarrolló varios periodos de investigación (años 1983, 1985 y 1987) en la comarca de Los Monegros. Su interés por los regadíos interiores españoles se amplió a los de la fachada mediterránea, dando lugar a una estrecha colaboración académica, en un primer momento, y de amistad después, con diversos profesores del departamento de Análisis Geográfico Regional de la Universidad de Alicante. Fruto de ello fue la participación de 
miembros de ambos departamentos en las Acciones Integradas Hispano-Italianas que se desarrollaron en 1989 y 1991, así como su integración en la red de movilidad de estudiantes (Programa Sócrates-Erasmus) y la estancia de profesores alicantinos en la Università degli Studi di Padova, cuya formación académica y personal se enriqueció con su docencia y amistad.

Su relevancia en la disciplina geográfica italiana y europea ha sido reconocida con numerosas distinciones académicas. Fue director del departamento de Geografía G. Morandini de la Università degli Studi di Padova (1999-2001) y del programa de doctorado Uomo e ambiente (1995-1999), componente de la Junta Directiva de la Associazione dei Geografi Italiani, responsable científico del Grupo de Trabajo de la Asociación de Geógrafos Italianos Assetti sociali, processi territoriali e confliti ambientali in Africa y miembro del Consejo Asesor de publicaciones italianas (Rivista di Studi Geografici, Rivista terra d'Africa, editada por la Universidad de Milán y Quaderni di Materiali, del Departamento de Geografía de la Universidad de Padua), españolas (Revista Investigaciones Geográficas de la Universidad de Alicante) y francesas (Revue Géographique des Pyrenées et du Sud Oest de la Universidad de Toulouse).

$\mathrm{Su}$ influencia y su recuerdo permanecerán siempre entre aquellos que lo conocimos y que tuvimos el privilegio de aprender de sus profundos conocimientos geográficos, disfrutar con su enseñanza y, sobre todo, de su amistad.

María Hernández Hernández 


\title{
ANÁLISIS DE LA SINIESTRALIDAD AÉREA POR CAUSA METEOROLÓGICA (1970-1999)
}

\author{
M. Carmen Moreno García \\ M. Ángel Gil Aguinaliu \\ Departamento de Geografía Física y Análisis Geográfico Regional \\ Universidad de Barcelona
}

\section{RESUMEN}

En este artículo se realiza un estudio sobre la influencia que han tenido los factores atmosféricos en la seguridad aérea, en el ámbito mundial, en el período 1970-1999. Como resultado del análisis se observa que la siniestralidad aérea por causas atmosféricas representa una pequeña fracción del total de accidentes y tiene además una clara evolución descendente en el tiempo. Las causas más importantes son el engelamiento o acumulación de hielo en alas y fuselaje, la cizalladura o variación brusca del viento en cortos espacios, la turbulencia y la baja visibilidad. En cada caso se intenta ver la dependencia estacional y sus causas unido a la incidencia del factor humano.

Palabras clave: siniestralidad aérea, causas meteorológicas, engelamiento, cizalladura, reventón, turbulencia, baja visibilidad.

\section{ABSTRACT}

This article is about the influence that have had the atmospheric factors in the air safety between 1970 and 1999 . The air sinistrality by these causes is a little part of the total accidents and her evolution has experimented a notable descent. The most important causes are icing in the wings, wind shear, turbulence and low visibility. In each case we will see the relation with the season and the causes and too the human factor influence.

Key words: air sinistrality, meteorological causes, icing, wind shear, downburst, turbulence, low visibility.

\section{Introducción}

El transporte aéreo es, hoy en día, el modo de transporte más rápido y seguro. Atrás quedan los tiempos en que volar era una peligrosa aventura, en la que se dependía de débiles y lentos aviones y de unas condiciones meteorológicas que con mucha frecuencia 
eran adversas, dada la baja altura a la que se volaba. Actualmente, gracias a los avances en materiales, motores a reacción e instrumentación avanzada volamos en aviones veloces y muy seguros y prácticamente bajo cualquier tipo de condiciones atmosféricas.

A pesar de todos estos avances el moderno avión sigue dependiendo, aunque en menor medida, de las condiciones del medio en que se mueve, - la atmósfera-, y por ello es necesario seguir investigando en el marco de la meteorología aplicada a la aviación y de la tecnología aeronáutica, para que el vuelo sea más seguro y eficiente, sea cual sea el estado atmosférico.

Este estudio se centra en ver la influencia que en las tres últimas décadas han tenido los factores atmosféricos en la seguridad aérea dentro del ámbito mundial y comprobar la mejoría que se ha experimentado en este campo, mediante el análisis de los accidentes aéreos por causas meteorológicas. Habría sido interesante hacer un estudio desde décadas anteriores (desde los años 30), en el que se habrían apreciado los cambios debidos a los grandes avances experimentados en el sector, pero los datos no eran tan abundantes y se ha optado por estudiar sólo el periodo 1970-1999, que corresponde ya a la era actual y más moderna de la aviación.

Desde la época de los años 30-40 hasta hoy han habido cambios muy notables en el campo de la seguridad aérea respecto a los factores atmosféricos, que han hecho bajar notablemente la siniestralidad y que son enumerados a continuación de forma resumida:

a) La aparición del reactor en la década de los 50 ha supuesto volar a alturas de unos $10000 \mathrm{~m}$, con lo que el avión, en vuelo de crucero, está fuera del alcance de gran parte de los fenómenos meteorológicos potencialmente peligrosos como las tormentas. De todas formas, el peligro de los fenómenos atmosféricos en cotas medias y bajas no se puede evitar, ya que en la fase de despegue y aterrizaje el avión se mueve en estos niveles de altura.

b) La aparición y mejora de sistemas radioeléctricos de guiado en ruta y aterrizaje que permiten volar en todo tipo de condiciones meteorológicas, así como la incorporación del radar meteorológico.

c) La mejora en la predicción e información meteorológica para la aviación.

Por otra parte, cabe destacar que los factores físicos no son los únicos que desencadenan un accidente por causas meteorológicas, sino que en muchos casos también interviene el factor humano. Por ello, no puede deducirse un modelo físico en el que basarse para hacer predicciones, ya que la influencia humana no se puede modelizar. Así pues, el estudio que se hará es, estadístico-descriptivo, en el cual las causas meteorológicas se tratarán como factor de riesgo y no como determinante del accidente, ya que, generalmente, este factor de riesgo va combinado con la actuación del piloto, estado del avión o sus componentes, problemática del tránsito aéreo y otros factores operativos. Por ello, se explicarán cuáles son los factores de riesgo más importantes que hay en la atmósfera y se verá en cada caso si hay alguna regularidad estacional. Para evitar estos riesgos es conveniente conocer la climatología aeronáutica de la ruta que se va a volar para tener una primera aproximación de los problemas que potencialmente podemos encontrar y que serán confirmados o descartados con el parte diario e información de otras tripulaciones (Linés, 1982).

Cuando se habla de accidente ha de entenderse el suceso asociado con la operación de un avión dedicado al transporte civil de personas o mercancías en el que, o bien el avión ha sufrido daños irreparables, o bien han habido muertos o heridos graves (definición basada en la de la OACI). 


\section{Datos de origen}

Los datos utilizados en el presente estudio se refieren al período 1970-1999 a nivel mundial y han sido extraídos de estadísticas oficiales, disponibles en sendas páginas WEB especializadas sobre accidentes aéreos, cuya fiabilidad viene avalada por la recomendación de la Escuela Técnica Superior de Ingenieros Aeronáuticos de la Universidad Politécnica de Madrid.

En este estudio se tienen en cuenta sólo los accidentes del sector de vuelos comerciales de línea, dejando fuera los vuelos militares y los vuelos privados. Como aviones de línea se consideran aquellos que tienen una capacidad de llevar un mínimo de 19 pasajeros. Se incluyen los modelos posteriores a la II Guerra Mundial, excepto el DC-3, que aún opera en algún lugar. En la base de datos usada se incluyen informaciones sobre accidentes ocurridos en la Europa del Este en época de Guerra Fría aunque a veces los datos que aparecen son escasos o incompletos.

\section{Fundamentos físicos básicos del vuelo}

El avión, en vuelo nivelado, está en equilibrio de fuerzas, es decir, el peso está compensado por una fuerza de sustentación que se produce en el ala y la resistencia ejercida por el aire se compensa con la fuerza de propulsión del motor.

De cara al análisis que se pretende es importante comprender cómo se genera la fuerza de sustentación sobre el ala, ya que buena parte de los agentes meteorológicos responsables de los accidentes aéreos inciden de forma directa sobre la fuerza sustentadora. Es por ello por lo que se incluye este apartado donde se presentan de forma resumida los principales fundamentos teóricos en relación con el tema. Determinados fenómenos meteorológicos pueden modificar condiciones aerodinámicas y aún provocar una pérdida de sustentación e, incluso en otros casos, una pérdida de control del aparato provocándose así el accidente.

La sustentación se produce cuando el aire incide sobre el perfil alar a cierta velocidad, es decir, cuando el avión y el aire están en movimiento relativo (para que exista sustentación debe haber velocidad relativa suficiente entre aire y avión). Si sobre un ala se hace incidir una corriente de aire y se estudia la trayectoria del flujo alrededor de ésta, se obtiene un resultado como el que se observa en la figura $n^{\circ} 2$, en el que las líneas reflejan las trayectorias de las partículas del fluido, puestas de manifiesto con inyecciones de humo

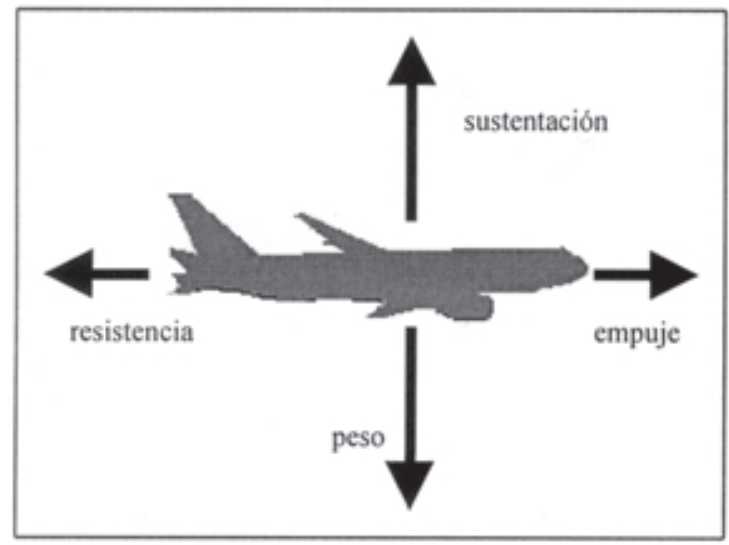

FiguRa 1. Fuerzas que actúan sobre el avión durante el vuelo. 


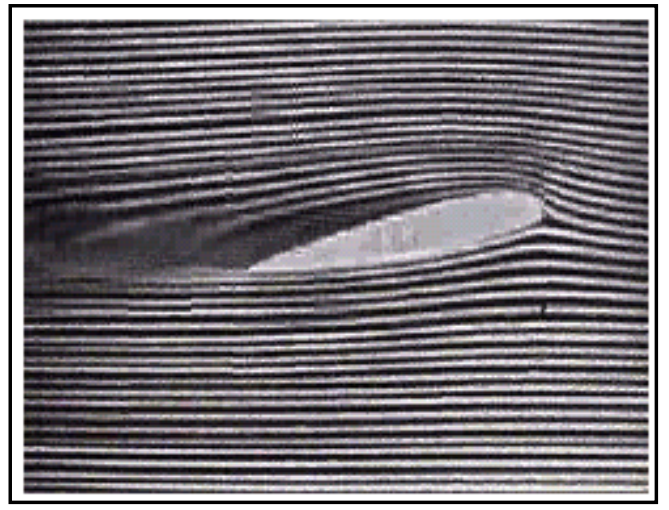

FIGURA 2. Flujo de corriente de aire sobre el perfil alar.

coloreado. Se puede apreciar que las líneas de corriente se aprietan en la parte superior del ala, mientras que en la parte inferior se ensanchan. Esto demuestra que la velocidad en la parte superior es mayor que en la inferior, lo cual trae como consecuencia una desigual distribución de presiones en ambas partes del ala, que da como resultado una fuerza de sustentación. Esto es así ya que, según el teorema de Bernoulli, en las zonas de mayor velocidad la presión es menor (parte superior), mientras que en las de menor velocidad (parte inferior) la presión es mayor. Por lo tanto, la presión inferior es mayor que la superior y fruto del desequilibrio se genera una fuerza neta que tira del avión hacia arriba, compensando a la fuerza peso. Aquella fuerza es la sustentación que permite al avión mantenerse en el aire mientras mantenga una velocidad mínima.

La fuerza de sustentación depende de los siguientes factores (Rosario, 1990):

a) la densidad del aire (I ),

b) la velocidad relativa aire-avión (V) (al aumentar V, aumenta la sustentación),

c) la superficie del ala $(\mathrm{S})$,

d) el ángulo formado entre el ala y la corriente del aire o ángulo de ataque (_) (al aumentar el ángulo, aumenta la sustentación), y

e) la forma del ala.

Experimentalmente se puede comprobar que la sustentación puede ser modelizada mediante la siguiente fórmula:

$$
\mathrm{L}=\frac{1}{2} / \mathrm{V}^{2} \mathrm{SC}_{\mathrm{L}}
$$

siendo $\mathrm{C}_{\mathrm{L}}$, un coeficiente que depende del ángulo de ataque y de la forma del ala, denominado coeficiente de sustentación. Puede decirse, a grandes rasgos, que al aumentar el ángulo aumenta $C_{L}$ hasta un valor máximo y si este ángulo se sobrepasa el avión pierde sustentación o entra en pérdida.

La sustentación en el avión puede ser alterada por cuatro causas:

a) por pérdida de la velocidad,

b) por aumentar el ángulo de ataque,

c) por alterarse la forma del ala o fuselaje (por ejemplo, por acumulación de hielo), variándose así el valor de $\mathrm{C}_{\mathrm{L}}$ al perderse las propiedades aerodinámicas, $\mathrm{y}$

d) por variación en la densidad del aire. 


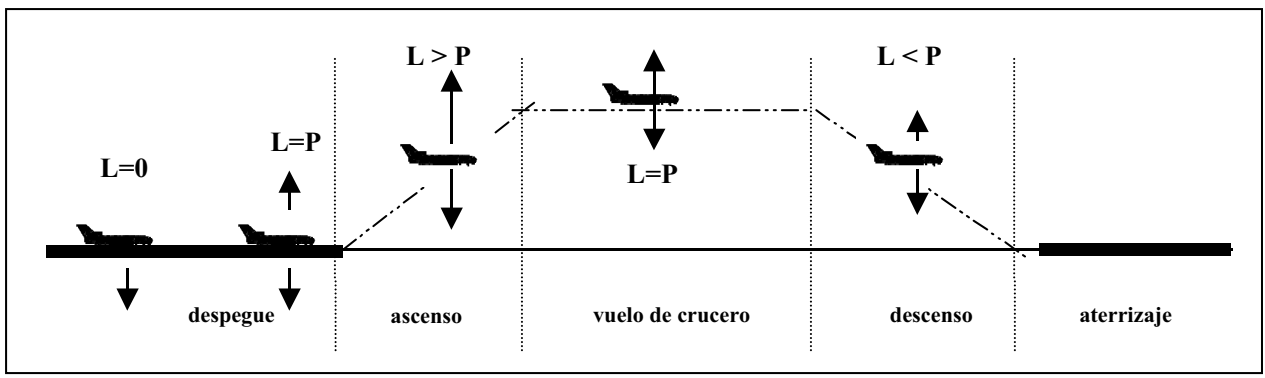

FIGURA 3. Relación entre las fuerzas de sustentación y peso en las diferentes fases del vuelo.

Sobre estos elementos actuarán los factores meteorológicos que provocan la mayor parte de los accidentes debidos a causas atmosféricas y que se analizarán de forma individualizada en cada caso. Los factores atmosféricos pueden actuar durante cualquier fase del vuelo, pero donde hay más peligro es en el despegue y aterrizaje, porque si nos encontramos con una disminución repentina de sustentación en estas fases críticas no se dispone de altura suficiente para corregir el problema y se puede producir un rápido impacto con el suelo. En cambio, en vuelo de crucero se tienen $10.000 \mathrm{~m}$ de altura para poder reaccionar y encontrar una solución.

En la figura $\mathrm{n}^{\circ} 3$ pueden observarse las fases que tiene un vuelo y la relación de fuerzas que intervienen en cada una de ellas. En el despegue, el avión aumenta la velocidad hasta que la sustentación iguala al peso (recuérdese que al aumentar $\mathrm{V}$, aumenta L). En el ascenso la sustentación es mayor que el peso y el avión asciende. El avión está en plena potencia y a alta velocidad. Esta fase puede ser muy peligrosa si aparece algún problema que afecte al equilibrio de fuerzas. En el vuelo de crucero, que suele discurrir en torno al nivel de los 10.000 metros, la sustentación iguala al peso y hay equilibrio de fuerzas. En el descenso el peso es mayor que la sustentación y el avión pierde altura para iniciar el aterrizaje. Ésta vuelve a ser una fase crítica, ya que se vuela a poca altura y cualquier adversidad se convierte en un grave peligro.

Los factores meteorológicos podrán incidir de forma diferente en función de la fase en la que actúen. Una vez conocidos estos principios básicos del vuelo se comprenderá mejor el análisis de la siniestralidad aérea causada por los distintos agentes o factores meteorológicos.

\section{Evolución general de la siniestralidad aérea (1970-99)}

La siniestralidad aérea ha descendido mucho en las últimas décadas. En la figura $n^{\circ} 4$ se puede apreciar que el número absoluto de accidentes ha descendido desde unos 70 al año en los setenta hasta alrededor de 40 a finales de los noventa. No obstante, al hacer un cálculo de accidentes totales por década se ve que de los años 70 a los 80 hay un descenso de 606 a 434 accidentes, pero entre los 80 y los 90, en cambio, hay un leve ascenso hasta 483. A pesar de ello puede afirmarse que la siniestralidad ha bajado notablemente, ya que, como se puede observar, el número de despegues o viajes al año ha crecido espectacularmente desde los 6 millones en 1970 hasta 18,7 en 1999. Así, la siniestralidad relativa o número de accidentes por millón de despegues ha bajado mucho en estas tres últimas décadas. En términos relativos hemos pasado de 11,5 accidentes /millón de despegues a un nivel de 2,3 accidentes por millón, lo cual supone que la siniestralidad ha descendido en un $80 \%$ en estos 30 años. 


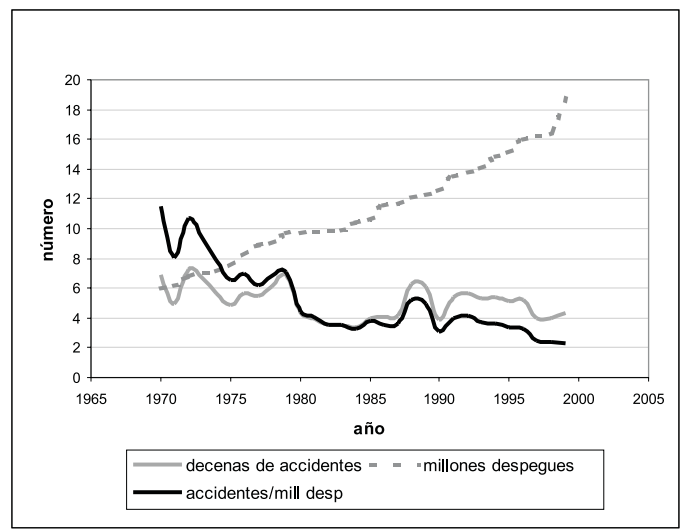

FIgURA 4. Evolución de la siniestralidad aérea total entre 1970 y 1999.

¿Qué fracción de estos accidentes es originada por causas meteorológicas? ¿Cuáles son los factores meteorológicos más peligrosos para la moderna aviación comercial?, etc. A continuación el análisis se centrará en estos aspectos y en otros, relacionados con lo que se podría denominar como una climatología de la siniestralidad aérea.

En la figura $n^{\circ} 5$ se observa que en estas tres décadas los accidentes por causas atmosféricas han sido una fracción muy pequeña, por lo que puede decirse que este tipo de factores son los menos probables a la hora de producirse un accidente. El mayor número de accidentes se deben a otras causas como fallos mecánicos, de estructura, humanos, etc. En los años 70 el 9,5\% de los accidentes eran por causas meteorológicas, mientras que en los 90 este índice ha bajado al 3,9\% (hay una probabilidad entre 11.000 .000 de que el mal tiempo sea el responsable de un accidente aéreo). En la figura $n^{\circ} 6$ puede comprobarse, en relación a esta pequeña fracción de accidentes, esta tendencia claramente descendente. Hay que señalar que la siniestralidad ha ido oscilando de un año a otro de forma irregular, pero

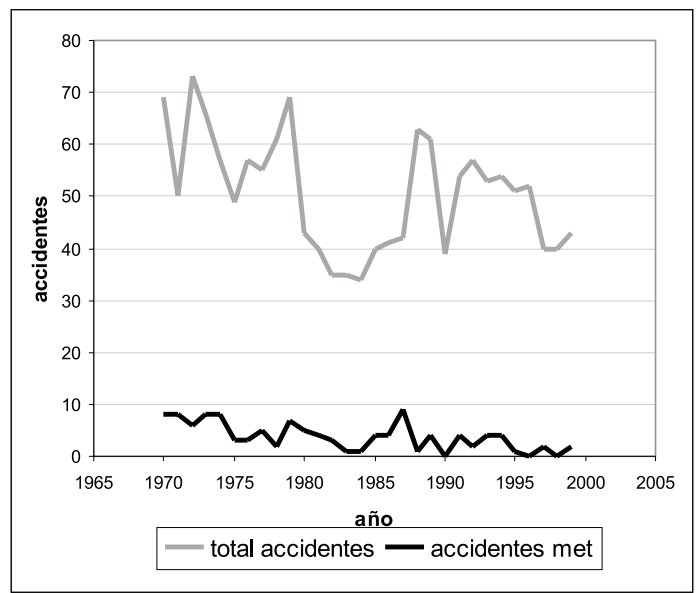

FIGURA 5. Comparación entre el número total de accidentes y los provocados por causas meteorológicas. 


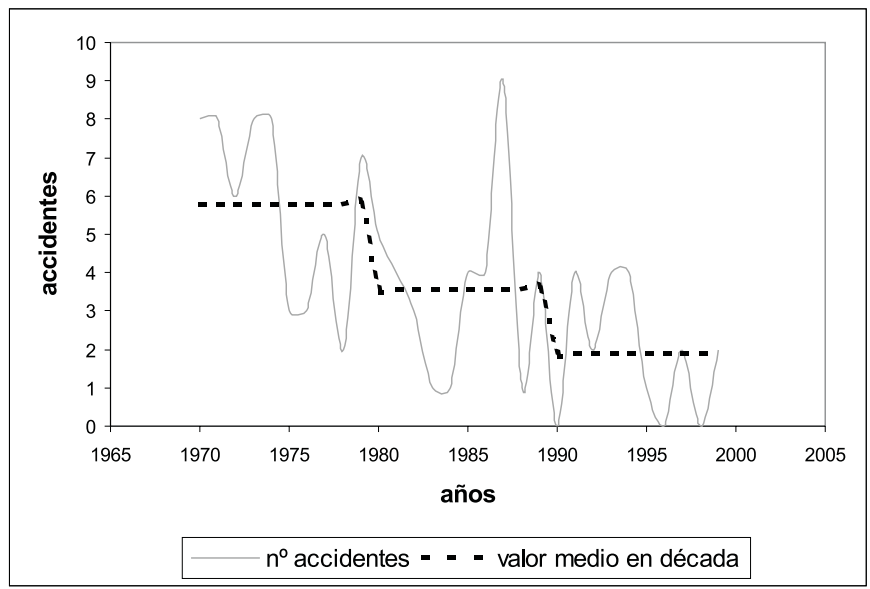

Figura 6. Evolución del número de accidentes por causas meteorológicas.

con tendencia a la baja. En la figura puede verse la irregularidad de esta variación instantánea, que observada en valor medio por década, se muestra con un decrecimiento continuo y, por ello, la tendencia global es de descenso de siniestralidad. La oscilación instantánea indica claramente que no hay un modelo matemático de variación, sino que existe una fuerte componente aleatoria, ya que a la regularidad atmosférica, debe añadirse la probabilidad del error humano que no es fácilmente matematizable. Así, aparecen estas irregularidades, aunque como se ha dicho con tendencia a la baja.

De los resultados obtenidos puede suponerse que, como la atmósfera no ha variado en comportamiento, sí que lo ha hecho la formación de los pilotos en este campo (factor humano) y los avances tecnológicos para la predicción y prevención en vuelo (radar meteorológico, partes meteorológicos, etc.). Aunque se haya mejorado mucho es muy importante conocer cuáles son los problemas reales con los que se encuentran los pilotos de hoy en día en relación con los factores atmosféricos. Por esta razón, en el siguiente apartado se analizarán una a una, las causas que contribuyen en mayor grado a la siniestralidad de origen atmosférico y meteorológico.

\section{Análisis de la siniestralidad aérea (1970-1999) por causa meteorológica}

En la figura $n^{\circ} 7$ se recoge la distribución, en números absolutos, de los accidentes aéreos producidos por diferentes causas de tipo meteorológico, señalándose, además, dentro de cada barra, el número de accidentes por década. Las causas, por orden de importancia, son:
a) el engelamiento o formación de hielo que se produce sobre el avión;
b) la cizalladura o variaciones bruscas en el viento en niveles bajos, que afectan notablemente a las maniobras de despegue y aproximación (wind shear);
c) la turbulencia o perturbaciones atmosféricas en forma de remolinos, que pueden hacer peligrar la estabilidad y estructura del avión (turbulencia mecánica, orográfi- ca, térmica, ondas atmosféricas y turbulencia en aire claro o TAC);
d) la baja visibilidad en niveles bajos; y
e) otras: el impacto de rayos, la turbulencia por estela de otros aviones y las ondas de montaña.




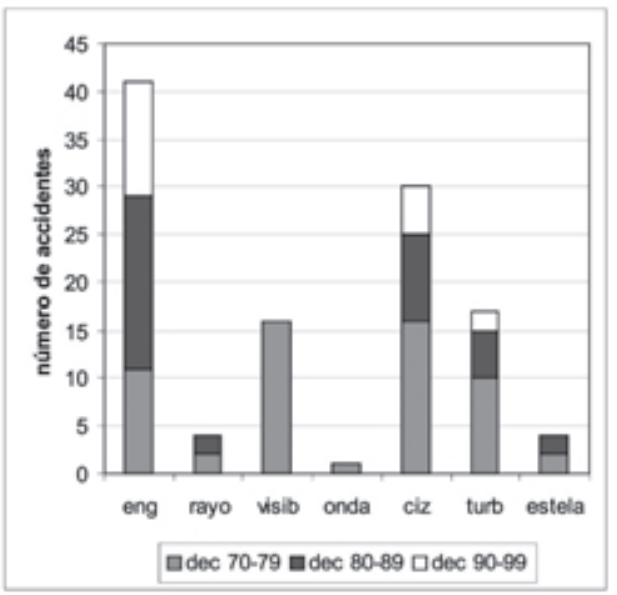

FIGURA 7. Distribución de las principales causas en los accidentes por factores meteorológicos.

Respecto a la evolución temporal puede decirse que excepto el número de accidentes por causa del engelamiento, que se mantiene, los debidos al resto de causas meteorológicas han descendido paulatinamente e, incluso, algunas prácticamente han desaparecido, lo cual confirma esta tendencia general a la baja.

Por ejemplo, los accidentes provocados por la baja visibilidad han sido los que más espectacularmente han bajado como puede observarse en la figura 7 . Le siguen en esta tendencia las causas de la cizalladura y turbulencias, que aunque no se hayan erradicado, han bajado notablemente y, para finalizar, los rayos, estelas de otros aviones y ondas de montaña, que ya no aparecen en estas últimas décadas y representan unos porcentajes marginales. El engelamiento es en conjunto la causa principal de accidente por causas atmosféricas, y se ve que no ha descendido ya que ha pasado en estas décadas por los siguientes valores: 11,18 y 12 accidentes, respectivamente. Es el caso más importante y el que se estudiará con mayor detalle.

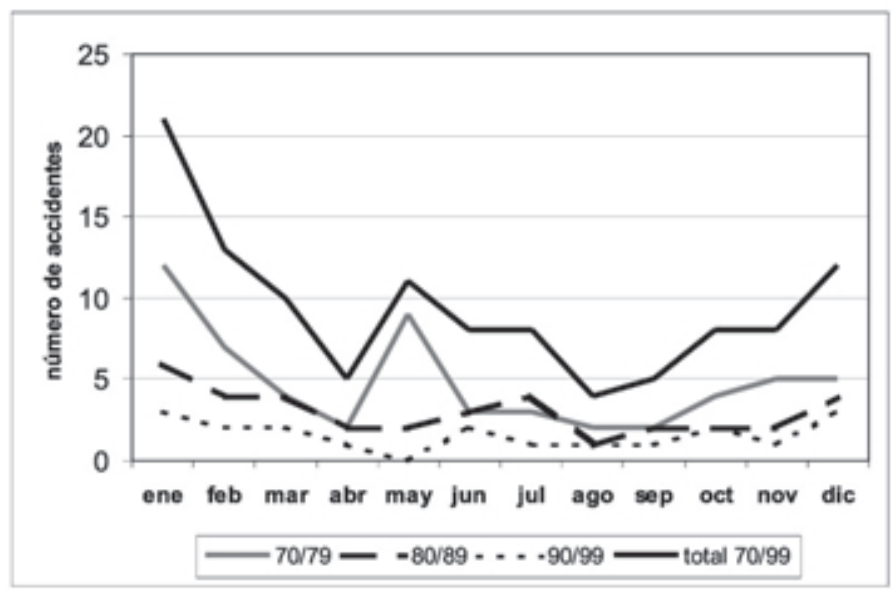

FiguRa 8. Distribución, por meses y décadas, de accidentes aéreos debidos a causas meteorológicas (1970-1999). 


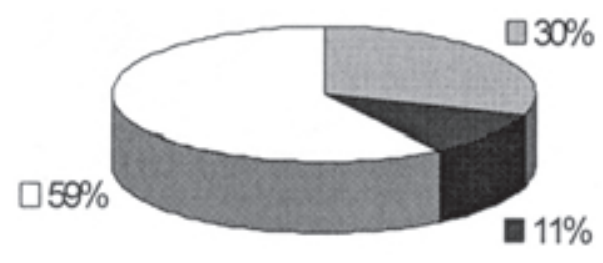

$\square$ DESPEGUE $\square$ CRUCERO $\square$ ATERRIZAJE

FIGURA 9. Distribución de los accidentes por causas meteorológicas en las diferentes fases del vuelo.

Otro aspecto interesante es la estacionalidad, o sea, ver cuáles son los períodos más propensos a la existencia de un accidente por causas atmosféricas. En la figura $\mathrm{n}^{\circ} 8$ puede verse que hay dos períodos importantes: los meses invernales (diciembre, enero y febrero) y los meses de mayo, junio y julio. En el primer grupo, el máximo se da en el mes de enero y en el segundo, en el mes de mayo. De todas formas, este máximo de mayo en las tres décadas es un tanto artificial, pues si se ven las gráficas estacionales de las tres décadas por separado se comprueba que el máximo de mayo sólo aparece en la primera (1970-79), desapareciendo después. Por lo tanto, puede atribuirse esto a una anomalía de esta década, pues las causas que aparecieron en mayo de esa época se han reducido notablemente e incluso han desaparecido en esta última década. También hay que destacar que el número de accidentes por mes ha ido descendiendo en cada década, llegándose a la última (90-99) con un mínimo absoluto de accidentes en los meses centrales del año y un máximo en los meses invernales, alrededor de enero. Más adelante, al analizar cada tipo de accidente por separado, habrá que estudiar el origen de esta distribución mensual, ya que al ver la estacionalidad de cada tipo de accidente, se podrá entender así la contribución espectral de cada uno a esta distribución mensual.

Otro aspecto interesante es ver la incidencia de los fenómenos meteorológicos en las diferentes fases del vuelo. En la figura $n^{\circ} 9$ se representa la distribución de los accidentes meteorológicos ocurridos en las diferentes fases del vuelo, comprobándose que el menor porcentaje de accidentes corresponde a la fase de crucero, con un 11\%. Esto es así, ya que, como se ha dicho anteriormente, en los aviones actuales se vuela a unos 10.000 metros y en esta zona se está a salvo de la mayoría de los fenómenos atmosféricos peligrosos para el vuelo como pueden ser las tormentas, el granizo, el engelamiento fuerte, etc. Este factor de la altura ha sido uno de los que más ha contribuido para dotar al vuelo de una mayor comodidad y seguridad. Pero hay dos fases que no escapan a las incomodidades atmosféricas: son las fases de despegue y aterrizaje, donde se da el mayor porcentaje de accidentes. El mayor peligro está en la fase de aterrizaje, con un 59\% de los accidentes, mientras que en la fase de despegue se produce el $30 \%$. Por lo tanto, desde el punto de vista meteorológico la fase más delicada es el aterrizaje, ya que es cuando el piloto debe actuar con la máxima precisión, puesto que cualquier problema relacionado con la visibilidad, turbulencia, cizalladura, engelamiento estructural, etc., puede poner en peligro el éxito de la maniobra. 


\subsection{Los accidentes aéreos por engelamiento}

El engelamiento, como ya se ha dicho, es la principal causa meteorológica de accidente aéreo $(36 \%)$ en estos últimos 30 años y, además, es la única que ha mantenido la cuota de accidentes a lo largo del tiempo, lo cual indica que probablemente sea el problema al que mayor atención deba prestarse. Se denomina engelamiento a la formación de hielo que se produce en el avión bajo determinadas circunstancias (Ledesma, 1993). Para que se produzca es necesario que el avión pase por una zona de nubes o precipitación, en la que las gotas de agua estén en estado de subfusión. Al pasar el avión por estas zonas, el agua se congela instantáneamente sobre las alas, los motores y el fuselaje del mismo, produciéndose el engelamiento. Éste se da en torno al nivel altitudinal de la atmósfera que está a $0^{\circ} \mathrm{C}$ - la línea isocero- . Al disponer de mapas que contienen esta información se pueden localizar las zonas de potencial engelamiento. Por ejemplo, puede decirse que, en invierno, en nuestra península, la línea isocero está a nivel de suelo y en verano está sobre los 4.000 $\mathrm{m}$ de altura.

El hielo que se puede formar es de diferentes tipos:

a) hielo granular que pesa poco y se desprende con facilidad;

b) hielo claro que es el tipo más importante. Es denso, transparente y se desprende con dificultad. Se produce con grandes gotas subenfriadas o lluvia engelante entre los $0^{\circ}$ $\mathrm{y}-10^{\circ} \mathrm{C}$;

c) por adherencia de nieve húmeda; $y$

d) escarcha formada sobre la superficie del avión, generalmente en invierno, cuando el avión ha permanecido por la noche en tierra a la intemperie. En este caso es necesario eliminar la escarcha antes de volar, habitualmente con medios químicos. A este tipo de engelamiento se le llama engelamiento en tierra y constituye el más importante en la estadística de accidentes. Este tipo de accidentes están provocados, en general, por errores humanos, al no eliminar esta fina capa helada. En algunos casos, también se puede formar en vuelo.

La acumulación de hielo en el avión depende de la forma y la velocidad de éste, así como de la altura de vuelo. Las formas anchas sin borde, por ejemplo, recogen menos hielo. O la mayor velocidad de los modernos aviones que hace que, por el doble efecto de un mayor calentamiento por rozamiento con el aire y la permanencia de menos tiempo en la zona engelante, el riesgo de engelamiento sea menor. Durante muchos años el engelamiento en vuelo fue un grave problema, pero hoy día ya no, porque los aviones disponen de buenos equipos antihielo, no obstante el engelamiento en tierra sí que es todavía preocupante. Si el avión vuela muy alto, el engelamiento es muy improbable, dado que la nubosidad es prácticamente nula. En estas zonas, además, la temperatura es tan baja, que hace también difícil el engelamiento fuerte.

Existe una escala que clasifica el engelamiento en diferentes grados: ligero, moderado o fuerte, según la velocidad de acreción de partículas de hielo al aparato. Con engelamiento fuerte se pueden llegar a tener fuertes vibraciones que afectan a los motores e, indirectamente, a los mandos y control del aparato. Los efectos del engelamiento pueden afectar también al rendimiento del avión, contribuyendo a su disminución, al adherirse el hielo a los motores y reactores. Si esto ocurre en las fases críticas se puede tener una pérdida de potencia y sustentación que dé lugar al impacto con el suelo. También se puede adherir en el borde de ataque de las alas y en la cola siendo éste el efecto más importante y peligroso. $\mathrm{Al}$ variar la forma de estos elementos, se empeora el $\mathrm{C}_{\mathrm{L}} \mathrm{y}$ el avión pierde sustentación y aumenta resistencia, con lo cual, si se vuela a baja altura, es probable el impacto. Por ejemplo, si se intenta despegar con escarcha en las alas es probable que no se tenga el 


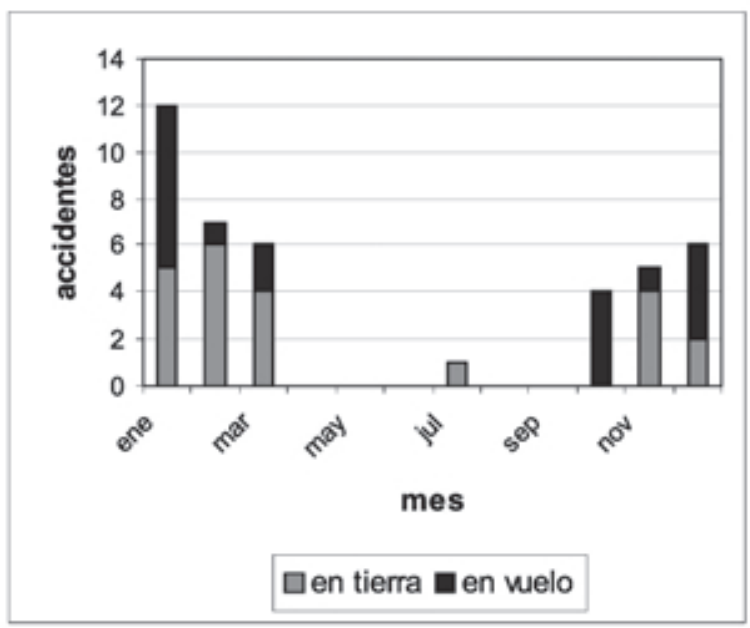

FIGURA 10. Distribución mensual de los accidentes por engelamiento.

empuje necesario para elevarse e iniciar el vuelo. Este tipo de accidente es el más frecuente dentro de los atribuidos al engelamiento siendo, en muchos casos, a causa de errores en la revisión previa al vuelo (factor humano). El hielo en cola produce vibraciones que pueden ser muy serias. Otro efecto importante es que al acumularse hielo hay más peso sobre el avión y se requiere un empuje mayor.

Asimismo el hielo se puede adherir en las hélices, perdiéndose el rendimiento hasta tener una alta probabilidad de pérdida, o en los tubos de Pitot, con el falseo en las medidas de velocidad, altitud o régimen de ascenso, o en la antena, el tren de aterrizaje y el limpiaparabrisas.

Para combatir el engelamiento los aviones disponen hoy día de medios mecánicos, que resquebrajan el hielo en las alas, medios térmicos que calientan aquellas partes que se quieren proteger (pueden usarse resistencias eléctricas, que aumentan la temperatura evitando el engelamiento o aprovechar el calor de los gases de escape de los motores) y de medios químicos, que licúan el hielo formado.

En los modernos aviones, los medios de que se dispone son suficientes, pues, para que no se den excesivos problemas, siendo muchos de ellos atribuibles al error de la tripulación, sobre todo, en el engelamiento producido antes del despegue. En los 41 casos observados puede atribuirse fallo humano a 22 de ellos, lo cual supone que en este grupo el factor humano es, ciertamente, muy importante, una vez que se dan las condiciones de engelamiento. La figura $\mathrm{n}^{\mathrm{o}} 10$ muestra la estacionalidad de los accidentes por engelamiento, observándose que los meses de mayor peligro, como era previsible esperar, son los de otoño e invierno. El peor mes es el de enero, seguido por los meses de febrero, marzo, diciembre, noviembre y octubre. En los meses de primavera y verano el riesgo es nulo. No obstante, en el mes de julio aparece un caso que no se puede considerar como verano, ya que corresponde a Nueva Zelanda (hemisferio Sur). Es normal que el fenómeno se dé en otoño e invierno que es cuando se registran las temperaturas más bajas y más bajos niveles de altura de isocero que provocan mucho más riesgo de engelamiento en vuelo a bajos niveles.

Se han representado en el gráfico dos tipos de engelamiento: el engelamiento en vuelo, que es el provocado al paso del avión en vuelo por la zona engelante, y el engelamiento en tierra ya comentado. Ambos tipos se reparten casi por igual, por lo que parece difícil 
extraer conclusión alguna, ya que en los accidentes debidos al engelamiento en tierra, además predomina el factor humano.

Finalmente, hay que indicar que el engelamiento afecta predominantemente a las fases de despegue (22 casos) y aterrizaje (17 casos), mientras que el engelamiento en vuelo solamente supone un $5 \%$ de los casos (2 casos). Esto viene a apoyar la idea expuesta anteriormente de que el engelamiento se produce, sobre todo, a niveles de baja altura.

\subsection{Los accidentes aéreos por cizalladura}

Esta es la segunda causa de siniestralidad meteorológica con un 26,5\% de los casos, pero a diferencia de la anterior, aquí, la tendencia, por décadas, es a la baja, ya que se pasa de 16 casos en la década de los 70 a 9 y 5 casos, respectivamente, en los años 80 y 90 . Se observa pues una evolución muy positiva, que puede ser debida a un conocimiento más amplio por parte de los científicos en la comprensión del fenómeno, a una mayor experiencia en las tripulaciones, a la mejora de la predicción y a la utilización del radar meteorológico para aquellos casos de cizalladura por la acción de núcleos tormentosos. Este fenómeno es fuente de accidentes a niveles bajos (hasta una altitud de unos $500 \mathrm{~m}$ ), donde la altura para maniobrar es poca y hay riesgo de accidente por cualquier alteración en las condiciones de vuelo. Por lo tanto es un fenómeno que actúa en niveles de aterrizaje y despegue, habiendo ocurrido en estas fases en el $80 \%$ y el $20 \%$ de los casos contabilizados, respectivamente.

La cizalladura (wind shear, en inglés) se define como un cambio brusco de la velocidad del viento sobre una corta distancia, pudiéndose dar esta variación tanto en el módulo de la velocidad como en su dirección Estas bruscas variaciones influyen en un alto grado en los grandes y pesados aviones a reacción de pasajeros en el transcurso de las operaciones de aproximación y despegue, pudiéndose llegar a situaciones críticas de desastre aéreo. En situaciones excepcionales se han llegado a observar cambios de $180^{\circ}$ en la dirección y de 50 nudos en la velocidad. El mecanismo del wind shear sobre el avión es el siguiente:

a) si el viento variase muy lentamente de módulo o dirección, la velocidad del avión se iría adaptando de tal forma que la velocidad relativa respecto al aire fuese constante. Se produce entonces un efecto de deriva.

b) si el viento varía muy rápidamente, el avión no puede variar tan rápido su velocidad debido a la fuerte inercia y nota una brusca variación transitoria en la velocidad relativa viento-avión que afecta a la sustentación. Éste es el efecto del wind shear. Las situaciones más importantes que pueden darse son:

- racha aparente de cara: se produce cuando el viento está de cara y aumenta bruscamente, entonces el avión nota automáticamente un aumento en la velocidad de cara y así, la sustentación aumenta también bruscamente de forma transitoria. Cuando ocurre esto el avión se eleva bruscamente de su senda de aproximación o despegue. Esto puede suceder también si el viento es de cola y pierde fuerza bruscamente (figura $\mathrm{n}^{\circ} 11$ ).

- racha aparente de cola: se produce cuando el viento está de cara y de repente disminuye, entonces el avión nota asimismo automáticamente una disminución en la velocidad de cara y así, habrá una brusca y transitoria pérdida de sustentación. Cuando esto ocurre el avión pierde altura de forma muy brusca y si está cerca del suelo, puede darse el impacto. Esto puede suceder también si el viento es de cola y aumenta bruscamente de valor (figura $\mathrm{n}^{\circ} 12$ ).

Estos efectos son muy peligrosos a baja altura, ya que una pérdida de altura a estos niveles y de forma brusca lleva consigo una alta probabilidad de accidente. En cambio, a 


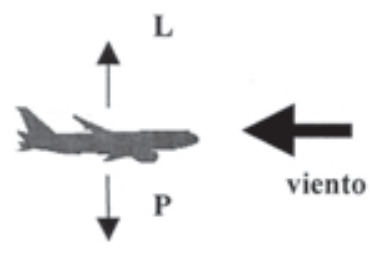

$\mathbf{L}=\mathbf{P}$

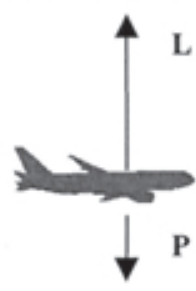

$\mathbf{L}$

$\mathbf{L}>\mathbf{P}$

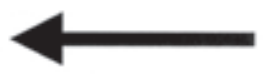

viento

RACHA DE CARA: Al aumentar la velocidad aumenta la sustentación y el avión asciende bruscamente

FiguRa 11. Efecto de la racha de cara por efecto de wind shear. Tendencia del avión al ascenso.

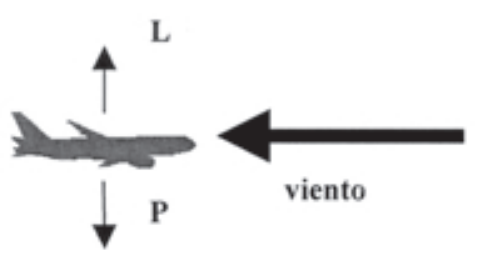

$\mathbf{L}=\mathbf{P}$

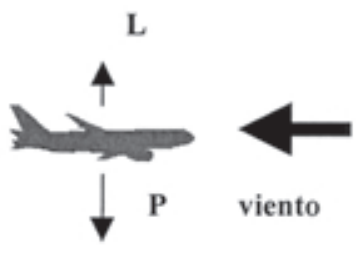

$\mathbf{L}<\mathbf{P}$

RACHA DE COL_A: Al đisminuir la velocidad disminuye la sustentación y el avión cae bruscamente

FIGURA 12. Efecto de la racha de cola por efecto de wind shear. Tendencia del avión al descenso.

gran altura se dispone de espacio suficiente para una rectificación. Puede haber, asimismo, rachas aparentes de costado que provocan efectos de desequilibrio en el alabeo del avión, pudiendo haber impacto con el suelo con una de las dos alas.

La cizalladura puede originarse por distintas situaciones o condiciones meteorológicas (Ledesma y Baleriola, 1993):

a) por actividad tormentosa,

b) por el paso de frentes y líneas de turbonada,

c) por corrientes en chorro (Jet Stream),

d) por brisas y alisios,

e) por rozamiento en la capa límite planetaria,

f) por obstáculos, y

g) chorros de bajo nivel altitudinal.

El caso de las tormentas es el más importante y peligroso y consiste en la existencia de fuertes corrientes o rachas descendentes bajo el núcleo de la nube tormentosa que inducen la cizalladura, especialmente en el inicio de la fase de madurez de la tormenta. Estas rachas o reventones pueden ser de muy diversas escalas pero son especialmente importantes los correspondientes a la microescala, en la cual, el reventón o microráfaga (microburst) queda definido como una intensa y localizada corriente vertical descendente de muy poco diáme- 


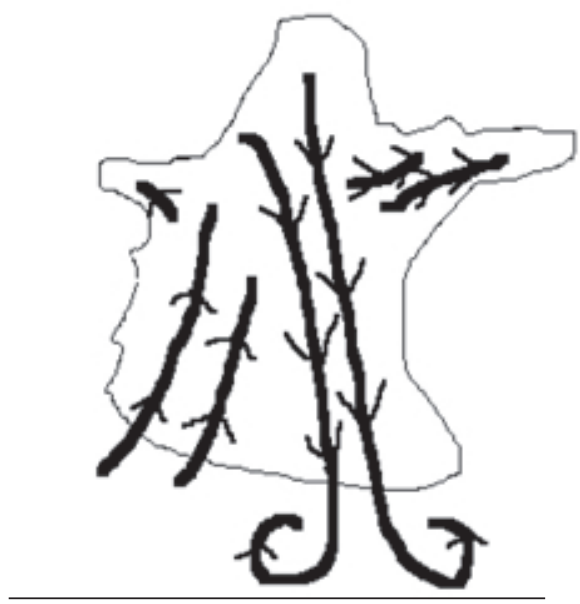

Figura 13. Downburst y outburst en el modelo de tormenta de Fujita. (Fuente: Ledesma, 1993).

tro y muy corta vida (Ledesma, 1993). Estas ráfagas, al impactar con el suelo se extienden con violencia y pueden originar torbellinos curvilíneos de eje horizontal (figura 13). Estas rachas tienen los nombres de reventón descendente (downburst) y expansivo (outburst), respectivamente. Un avión, en la aproximación final, puede encontrar este sistema de downbursts y outbursts y quedar sometido, por efecto de rachas de cara, cola y descendentes, a ganancias y pérdidas de altura no deseadas que son peligrosas para la finalización de la maniobra. Actualmente el problema más grave se encuentra al iniciar una aproximación final o despegue en el justo momento en que la primera gran racha descendente de la tormenta, al iniciar el estado de madurez, llega al suelo. Los downdrafts pueden encontrarse también en tormentas secas y otras situaciones y no deben confundirse con los tornados que son de extensión y efectos similares pero de diferente estructura ya que en estos últimos las corrientes son ascendentes y en espiral (Linés, 1996). En las microráfagas el aire desciende a gran velocidad (6000 a 7000 pies por minuto) y el aire del outburst puede llegar a tener velocidades de $280 \mathrm{Km} / \mathrm{h}$. El diámetro es muy pequeño ( 1 a $3 \mathrm{Km}$ ), la vida es muy corta (hasta 15 minutos), el tiempo de reacción después del reconocimiento es de unos pocos segundos (hasta 5 segundos) y además los instrumentos de presión del avión no dan lecturas correctas. Con tales características no es de extrañar que su detección sea muy difícil o imposible (Cubero, 1996). En 1975 en el aeropuerto de Nueva York se produjo un grave accidente por esta causa con el triste balance de 113 víctimas; a raíz de aquel suceso se profundizaron los estudios de las estructuras tormentosas y los efectos de la cizalladura.

En los últimos años se han intentado métodos de medición de la cizalladura como el LLWAS consistente en una serie de anemómetros situados en los alrededores de aeropuertos, dispositivos de SONAR, de LÁSER y de DOPPLER, aunque las limitaciones son grandes especialmente por el corto espacio de tiempo que hay entre la detección y el aviso a la aeronave. Actualmente la casi totalidad de las aeronaves de gran capacidad llevan un sensor para detectar la cizalladura, que incluso puede conectarse al piloto automático para asumir un pilotaje asistido.

También puede producirse cizalladura por el paso de un frente, ya que al pasar la superficie frontal por un punto, como por ejemplo, un aeropuerto, encontramos una discontinuidad en el campo de viento y por ello, al paso de un frente, se darán situaciones de 


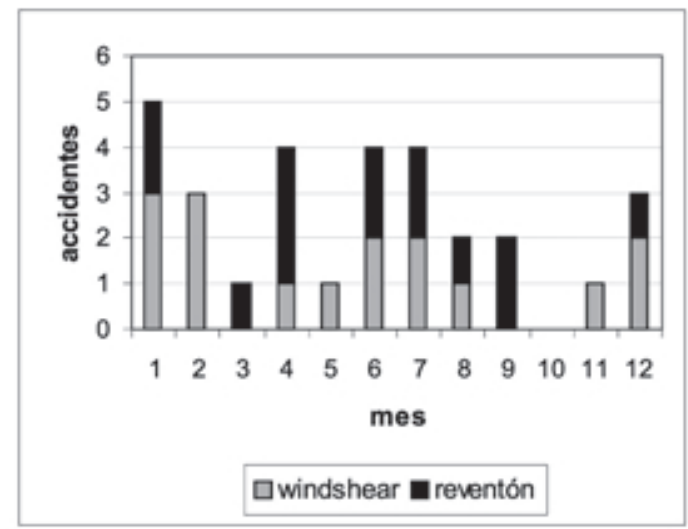

Figura 14. Distribución mensual de los accidentes por cizalladura o wind shear.

bruscos cambios en la velocidad con el consabido peligro. En los datos analizados se ha encontrado un caso de accidente al paso de un frente y otro al paso de una línea de turbonada.

Las corrientes en chorro de bajo nivel que constituyen corrientes de aire relativamente caliente por encima de una capa de aire frío y en calma también pueden producir wind shear. Estas corrientes suelen darse en noches claras con inversión térmica, pudiendo provocar una fuerte cizalladura.

Otras causas no tan importantes son: las brisas de mar y de montaña (que pueden causar fuerte cizalladura hasta una altura de $600 \mathrm{~m}$ ), los vientos alisios, el rozamiento en la capa límite planetaria (que produce cambios variables en la dirección del viento) y obstáculos diversos en las inmediaciones del aeropuerto, tales como hangares, montañas, precipicios, etc.. (que dan lugar a cambios vectoriales de viento).

La distribución mensual de los accidentes por cizalladura se muestra en la figura $\mathrm{n}^{\circ} 14$, donde se han representado los casos debidos a la actividad tormentosa (reventón) y también el resto de causas, agrupadas todas ellas. El máximo número de accidentes se da en el mes de enero, seguido de los meses de abril, junio y julio. En la cizalladura de origen tormentoso los casos se concentran en su mayoría alrededor del verano (junio, julio, agosto y septiembre), en invierno (diciembre y enero) y en primavera (marzo y, sobre todo, abril). En el resto de causas los máximos se dan en invierno también (diciembre, enero y febrero), seguido de otro núcleo en verano (junio y julio). El $80 \%$ de los casos, probablemente en aviones de instrumentación no muy desarrollada, ocurren en el aterrizaje, cobrando un gran protagonismo, por tanto, el factor humano, ya que se trata de un momento crítico en que se deja el vuelo automático y se cambia a manual para ejecutar la toma de tierra y es donde, a veces, por exceso de operaciones a realizar o por sorpresa pueden darse problemas de falta de reflejos de los tripulantes cuando se ven ante un inconveniente tan repentino y grave. La mejor prevención consiste en un buen entrenamiento de las tripulaciones y disponer de una buena información meteorológica.

\subsection{Los accidentes aéreos por turbulencia}

Los accidentes aéreos por turbulencia suponen un $15 \%$ de los casos totales. La turbulencia, al igual que la cizalladura, ha bajado muy notablemente en proporción, ya que ha pasado de 10 casos en los años 70, a 5 y 2 en los 80 y 90, respectivamente y esto supone 
un descenso del orden del $80 \%$ en estos 30 años. La turbulencia consiste en la aparición de movimientos desordenados en la corriente de un fluido que provocan la formación de perturbaciones en forma de remolinos. Según Ledesma (1981) los principales peligros de la seguridad en vuelo son en primer lugar la turbulencia, seguida del granizo, el engelamiento y las descargas eléctricas. Hoy día, se ve, con los datos analizados, que todos los factores citados, salvo el engelamiento, no ocupan ya los primeros puestos en el ranking de accidentes, lo que significa que dichos factores han de estar bien controlados.

La turbulencia consiste en que el avión pasa por zonas en las que encuentra remolinos de muy diversos tamaños y fuerzas. Las turbulencias pueden aparecer desde la escala sinóptica hasta la pequeña escala o microescala. Al entrar un avión en una zona de turbulencia se ve sometido a las ascendencias y descendencias que imprimen estos remolinos y así, en función de la intensidad de éstos, pueden producirse desde pequeños meneos a grandes sacudidas en la estructura. Hay que advertir que toda turbulencia produce cizalladura, pero no ocurre siempre a la inversa (Linés, 1982; Ledesma, 1981; Dole, 1984).

El avión al pasar por los remolinos sufrirá ascendencias y descendencias o bien sacudidas y vibraciones cuyo ritmo dependerá de la velocidad y tamaño de esos remolinos y de la velocidad del avión. En los aviones modernos, al ser la velocidad muy alta, el número de remolinos que pasa por unidad de tiempo es muy alto y así el avión está sometido a esfuerzos hacia arriba y abajo con alta frecuencia, produciéndose vibraciones que pueden hacer peligrar la estructura del avión. La turbulencia también es peligrosa en las maniobras de aterrizaje y despegue, por la obvia proximidad al suelo y asimismo, puede ser importante en niveles altos (TAC). En casos extremos la estructura del avión puede sufrir resonancia si la vibración de la estructura coincide con su frecuencia natural, pero esto se evita variando la velocidad de vuelo por la turbulencia.

La turbulencia puede ser según su intensidad: ligera (cuando los objetos del avión aún están en reposo), moderada (cuando el pasaje puede ser lanzado ocasionalmente fuera de los asientos), fuerte (cuando el avión puede quedar fuera de control) y extrema (con imposible control del avión). El origen de la turbulencia puede ser por causas mecánicas (debido a obstáculos), térmicas (debido a la inestabilidad del aire), por actividad tormentosa, por efectos orográficos (como las ondas de montaña) y por flujos veloces en altos niveles próximos a la tropopausa (ej.: corriente en chorro).

En cuanto a la distribución mensual de los accidentes aéreos causados por la turbulencia, se observa que a excepción de mayo, junio y octubre, el número de casos por mes es

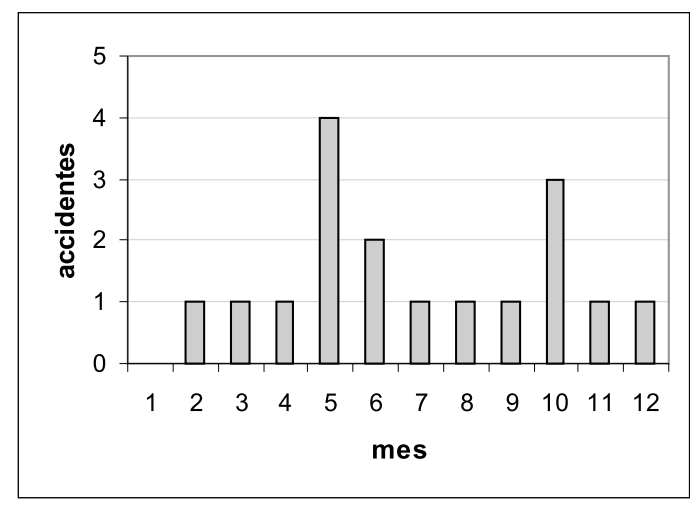

FIGURA 15. Distribución mensual de los accidentes por turbulencia. 
sensiblemente constante (figura $\mathrm{n}^{\circ} 15$ ). Se deduce pues que no hay relación de estacionalidad, ya que prácticamente en cualquier mes es probable encontrar turbulencia. Las anomalías de mayo y octubre ocurren en la primera década, mientras que en la de los 90 todos los meses se comportan de la misma forma. Por lo tanto podríamos decir que estos picos son debidos a causas aleatorias. Un hecho importante a destacar, en este tipo de accidentes, es que hay un gran porcentaje que ocurren en vuelo de crucero (35\%). En estos casos el accidente se ha producido por fallos estructurales en el avión. Así pues, la turbulencia es prácticamente el único fenómeno del cual no podemos escapar aunque vayamos a gran altura. Lógicamente, también hay casos por turbulencia en el despegue (18\%) y en el aterrizaje (47\%). Otro hecho destacable al analizar las estadísticas es que aproximadamente la mitad de los casos de turbulencia que eran fuertes, han sido a causa de los efectos de las tormentas que generan altos niveles de turbulencia. Hoy en día se evita circular por estas zonas gracias al radar meteorológico, que detecta con facilidad los núcleos tormentosos y las zonas de granizo y engelamiento.

\subsection{Los accidentes aéreos por baja visibilidad}

Los accidentes aéreos debidos a problemas de visibilidad representan un $15 \%$ del total, pero con la particularidad de que a partir de la década de los 80 ha habido un fuerte descenso de casos. Esto puede hacer pensar que estos accidentes han tenido una gran componente de error humano, factor éste que en los últimos años ha tenido que experimentar una notable mejora debido probablemente a la mejor preparación de las tripulaciones y en el uso de sistemas de aterrizaje instrumental y de modernos sistemas de balizas de pista. El $87 \%$ de estos accidentes ocurren en el aterrizaje, pudiéndose atribuir todos ellos, al error humano, al decidirse el aterrizaje por debajo de las condiciones mínimas de visibilidad. No se observa por ello estacionalidad en el fenómeno, ya que estos accidentes dependen exclusivamente del factor humano al no respetarse las reglas de procedimientos en baja visibilidad y haberse efectuado los aterrizajes, tanto visuales como instrumentales, por debajo de los límites de aeropuerto.

Para poder aterrizar en un aeropuerto se deben cumplir unos mínimos en los que intervienen la calificación del piloto, el tipo de avión y el tipo de ayuda en pista. La autorización de aterrizaje está en función de unos mínimos que dependen del alcance visual en pista (RVR) o distancia a la que se ven los objetos o luces en pista y de la altura de decisión, que es la altura a la que debe iniciar el piloto el procedimiento de aproximación frustrada si no ve el suelo. Aunque una aproximación se haga con la ayuda de instrumentos llega un momento en que se debe pasar a control manual y es aquí, al hacer la maniobra visualmente, donde se deben cumplir también los requisitos mínimos de aterrizaje, que son los que no han sido respetados por los pilotos. La excepción a esto son las llamadas categorías III A y III B en las que en teoría puede hacerse todo el proceso de aproximación y aterrizaje de forma automática aunque en general no suele autorizarse este procedimiento. La baja o insuficiente visibilidad puede originarse, principalmente, por la formación de nieblas, neblinas, calima y smog, y, también, por fuertes lluvias.

Hay que hacer notar que en este tipo de accidentes es difícil atribuir la causa y no es fácil delimitar la frontera entre el fallo humano, el debido a problemas de instrumentación y el causado por la propia visibilidad. Es por ello por lo que en este apartado no se han incluido otro tipo de accidentes en ambiente de baja visibilidad como colisiones en pista, en el aire o colisiones con montañas donde está permitido el vuelo o maniobras en condiciones de vuelo instrumental y donde presumiblemente el error es de tipo humano (tripulación o control aéreo) o también instrumental. 


\subsection{Otras causas}

Como casos marginales, aunque no por ello menos peligrosos, queda el impacto de rayos, la turbulencia de estela producida por circular muy cerca de la cola de otros aviones y las ondas de montaña, que conjuntamente suponen sólo el $8 \%$ de los casos.

El rayo consiste lógicamente en el impacto de tal elemento sobre la estructura del avión. Aunque el avión es metálico y por ello está aislado, no está exento de sufrir algunos efectos negativos de tal impacto, como daños estructurales graves, e incluso, chispas que al saltar pueden provocar incendios en los depósitos y motores, siendo por ello altamente peligroso.

La turbulencia de estela consiste en la turbulencia generada por grandes aviones (tipo JUMBO) al despegar y aterrizar, constituyendo un gran peligro para los aviones más pequeños que maniobran a continuación de éstos. Es, por este motivo, por lo que es muy importante respetar los intervalos necesarios de aterrizaje y despegue para evitar la turbulencia en estela (Ledesma y Baleriola, 1993; Dole, 1984). El 12 de noviembre de 2001 hubo en EEUU un accidente en el que muy probablemente la causa fue la generación de torbellinos en estela producidos por el despegue previo de un gran avión. En dicho accidente un Airbus 300 despegó 1'45" después de un 747 y se estrelló en una zona residencial con un balance de 265 muertos.

Las ondas de montaña consisten en un fenómeno turbulento muy poco probable en vuelo de crucero, pero muy peligroso. Se produce cuando flujos de aire muy veloces inciden perpendicularmente, bajo ciertas condiciones, sobre una barrera montañosa. Pueden originar turbulencias hasta el nivel de la tropopausa, siendo incluso de intensidad extrema (Linés, 1982). Actualmente estas ondas de montaña están creando problemas muy serios en los Andes.

\section{Conclusiones}

El número de accidentes aéreos en los cuales han sido determinantes los factores meteorológicos es una fracción muy pequeña del total, disminuyendo, además en promedio de forma continua durante las tres últimas décadas. La explicación radica en que cada vez se conocen mejor las causas atmosféricas y esto hace que las tripulaciones y la asistencia en tierra puedan prevenir y evitar estos riesgos cada vez con mayor eficacia. En el período analizado (1970-1999) las causas atmosféricas o meteorológicas que más han influido en los accidentes aéreos han sido, por orden de siniestros, el engelamiento o acumulación de hielo en alas y fuselaje, la cizalladura o variación brusca del viento en cortos espacios, la turbulencia y la baja visibilidad y ya, por último, a mucha distancia otros fenómenos como el impacto de rayos, las ondas de montaña y la turbulencia en estela.

El mayor número de accidentes aéreos por causa meteorológica se concentran en la fase de aterrizaje, seguido del despegue; en cambio el porcentaje más bajo ocurre durante el vuelo de crucero, ya que en esta fase el avión vuela a una altura suficiente para evitar la mayor parte de los riesgos potenciales. Finalmente, se ha observado también cierta estacionalidad asociada a la propia de algunos de los fenómenos meteorológicos. El mayor número de accidentes se concentra principalmente en los meses invernales (diciembre, enero y febrero) debido, en gran medida, a la influencia del engelamiento. En el caso de la cizalladura destacan sobre todo, los meses de verano, lo que es explicable quizá por la actividad tormentosa convectiva típica de estos meses. 


\section{Bibliografia y fuentes de datos}

CUBERO, R. (1996): «Aviació comercial: enlairaments i aterratges» en II Jornades de Meteorologia Eduard Fontserè (Meteorologia aeronàutica), pp. 69-80, ACAM, Barcelona.

DOLE, Ch.E. (1984): Teoría del vuelo y aerodinámica. Paraninfo, Madrid.

I.N.M. (2000): Guía de Meteorología Aeronáutica. Madrid.

HOUZE, R.A. (1993): Cloud Dynamics. Academic Press, San Diego.

LEDESMA, M. (1981): Turbulencia atmosférica. Paraninfo, Madrid.

LEDESMA, M. y BALERIOLA, G. (1993): Meteorología aplicada a la aviación. Paraninfo, Madrid.

LINÉS, A. (1982): Climatología aeronáutica. Iberia, Madrid.

LINÉS, A. (1996): «Meteorologia subsinòptica. Situacions adverses. Problemes i solucions» en II Jornades de Meteorologia Eduard Fontserè (Meteorologia aeronàutica), pp. 51-60, ACAM, Barcelona.

ROSARIO, A. (1990): Manual del piloto privado. Paraninfo, Madrid.

VAN SICKLE, N. (1985): Aeronáutica moderna. Paraninfo, Madrid.

http://aviation-safety.net/about.htm

http://airdisaster.com

\section{Agradecimientos}

A D. Alberto Linés Escardó por la revisión del manuscrito y las valiosas sugerencias efectuadas al mismo. 
